\title{
DISEASE CHARACTERISTICS, SOCIODEMOGRAPHIC ASPECTS AND COMORBIDITIES IN DIFFICULT-TO-TREAT RHEUMATOID ARTHRITIS: A TERTIARY CENTER EXPERIENCE
}

Letícia Serrano Sartori ${ }^{1, *}$, Daniele Hayashi ${ }^{1}$, Larissa Andrade Santiago de Oliveira ${ }^{1}$, Sonia Maria Alvarenga Anti Loduca ${ }^{1}$, Rina Dalva Neubarth Giorgi ${ }^{1}$, Roberta de Almeida Pernambuco ${ }^{1}$, Nathalia de Carvalho Sacilotto ${ }^{1}$, Lucas Victória de Oliveira Martins ${ }^{1}$

1.Instituto de Assistência Médica ao Servidor Público Estadual, São Paulo (SP), Brazil.

*Corresponding author: leh_ss@hotmail.com

\section{BACKGROUND}

Recently, the European Alliance of Associations for Rheumatology (EULAR) defined difficult-to-treat (D2T) rheumatoid arthritis (RA) patients as those that maintain disease activity and have already failed $\geq 2$ biological or targeted-synthetic DMARDs with different mechanisms of action (MOA). In addition to disease characteristics, sociodemographic aspects and comorbidities can contribute to the persistence of disease activity. We aimed to assess those contributing factors in an observational study.

\section{METHODS}

Sixty RA patients were enrolled and categorized with 24 D2T and 36 non-D2T patients. Disease characteristics, sociodemographic aspects and comorbidities were assessed. Statistical analysis was performed on continuous and categorical variables. Comparisons were made with appropriate tests ( $p$ value $<0.05$ was considered significant).

\section{RESULTS}

Results are shown in Table1. No differences were found in age, gender, education, working situation and economic classification. In disease characteristics, D2T group had a higher mean CDAI, SDAI and pain VAS, also, a higher frequency of glucocorticoid and tsDMARD current therapy and difference in number of failed b/tsDMARD with or without different MOA. When comparing comorbidities, D2T group had a higher BMI mean and frequency of fibromyalgia, depression and anxiety.

\section{CONCLUSION}

In the D2T group, obesity, pain and mood disorders were identified as contributing factors. These factors impact in disease activity, show the clinical complexity of RA and must be assessed when tailoring the therapeutic strategies for a personalized medicine.

\section{KEYWORDS}

Rheumatoid arthritis, Difficult-to-treat, Comorbidities. 
Table 1. Difficult-to-treat and non-D2T patient characteristics.

\begin{tabular}{|c|c|c|c|}
\hline Variables & D2T $(n=24)$ & Non-D2T $(n=36)$ & $\mathrm{p}$ value \\
\hline \multicolumn{4}{|c|}{ Sociodemographic } \\
\hline Age (mean + SD) & $56.90 \pm 7.95$ & $57.53 \pm 6.41$ & 0.76 \\
\hline Female (\%) & 95.8 & 91.7 & 0.64 \\
\hline \multicolumn{4}{|l|}{ Education } \\
\hline First grade $(\%)$ & 4.2 & 14.0 & \multirow{3}{*}{0.26} \\
\hline Second grade $(\%)$ & 25.0 & 33.4 & \\
\hline Higher grade (\%) & 70.8 & 52.6 & \\
\hline \multicolumn{4}{|l|}{ Working situation } \\
\hline Housewife (\%) & 20.8 & 27.8 & \multirow{3}{*}{0.80} \\
\hline Retired/disabled (\%) & 41.7 & 33.4 & \\
\hline Working (\%) & 37.5 & 38.9 & \\
\hline \multicolumn{4}{|l|}{ Economic class } \\
\hline A-B (\%) & 8.3 & 16.7 & \multirow{2}{*}{0.36} \\
\hline C-E (\%) & 91.7 & 83.3 & \\
\hline \multicolumn{4}{|c|}{ Disease characteristics } \\
\hline Disease duration (mean + SD) & $15.13 \pm 6.12$ & $12.31 \pm 8.03$ & 0.15 \\
\hline RF/ACPA presence (\%) & 91.7 & 94.4 & 0.56 \\
\hline CDAI (mean + SD) & $13.38 \pm 11.80$ & $6.92 \pm 6.13$ & 0.01 \\
\hline SDAI (mean + SD) & $18.11 \pm 13.94$ & $7.92 \pm 6.53$ & 0.02 \\
\hline Pain VAS (mean + SD) & $4.43 \pm 2.21$ & $3.14 \pm 2.20$ & 0.03 \\
\hline $\mathrm{HAQ}($ mean + SD) & $0.86 \pm 0.71$ & $0.93 \pm 0.79$ & 0.80 \\
\hline \multicolumn{4}{|c|}{ Current therapy } \\
\hline Glucocorticoid (\%) & 75.0 & 44.4 & 0.019 \\
\hline csDMARD (\%) & 70.8 & 83.3 & 0.25 \\
\hline bDMARD (\%) & 58.3 & 61.1 & 0.83 \\
\hline tsDMARD (\%) & 37.5 & 2.8 & 0.01 \\
\hline \multicolumn{4}{|c|}{ Failed DMARD } \\
\hline csDMARD & $2(0-3)$ & $1(0-4)$ & 0.60 \\
\hline Total b/tsDMARD (median) & $3(2-6)$ & $0(0-3)$ & 0.001 \\
\hline Different MOA b/tsDMARD (median) & $3(2-5)$ & $0(0-1)$ & 0.001 \\
\hline \multicolumn{4}{|c|}{ Comorbidities } \\
\hline Comorbidities presence (\%) & 100 & 72.2 & 0.004 \\
\hline Comorbidities (number) & $3(1-8)$ & $3(1-5)$ & 0.09 \\
\hline $\mathrm{BMI}($ mean + SD) & $29.31 \pm 5.17$ & $26.61 \pm 3.75$ & 0.02 \\
\hline Fibromyalgia (\%) & 25.0 & 2.8 & 0.013 \\
\hline Depression (\%) & 45.8 & 5.6 & 0.001 \\
\hline Anxiety (\%) & 45.8 & 5.6 & 0.001 \\
\hline
\end{tabular}

